\title{
THE INVESTIGATION OF THE HIERARCHICAL STRUCTURE OF THE INTERSTELLAR MAGNETIC FIELDS
}

\author{
Lazarian A.L. \\ P.N.Lebedev Physical Institute, U S S R
}

\begin{abstract}
A new method of investigation of the hierarchical structure of the interstellar magnetic fields is discussed. Synchrotron intensity fluctuations are used. Statistical characteristics of the irregular magnetic field are available. The method was successfully tested.
\end{abstract}

\section{Introduction}

It is well known, that magnetic field is very important for the processes of fragmentation of molecular clouds and processes of star formation. But it is rather difficult to investigate the magnetic field itself. The problem is that the field has a very complicated spartial structure. The interstellar magnetic field can be approximated by a superposition of a regular " $\mathrm{H}$ " and a random " $\mathrm{h}$ " magnetic fields. Correlation properties of " $h$ " determine the hierarchical structure of the interstellar magnetic fields on the scales much smaller than the size of the Galaxy. Using traditional methods it is impossible to determine correlation functions of the interstellar magnetic field. That is why a new method of investigation was developed [1].

The intensity of synchrotron radio emission depends on the number density of relativistic electrons and on the strength of the magnetic field component normal to the line of sight. In light of observational evidence for cosmic-ray isotropy one may start by assuming that relativistic electrons are distributed uniformly in the Galaxy. But the magnetic field is not homogeneous. Hence the synchrotron radiation observed in different directions also ought to be nonuniform. The problem of the determination of correlation properties of magnetic field is caused by the fact that the radiation is accumulated along the lines of sight. Different hierarchical structures of the interstellar magnetic field contribute to the resulting intensity. Under these conditions the information about statistical properties of a random magnetic field seemed to escape. Working in collaboration with Chibisov G.V. we have shown that it was not the case and it was possible to determine the correlation functions of the chaotic magnetic field on the basis of sycnhrotron fluctuations.

\section{Materials}

As it was shown back in 1980 [2] a correlation function of intensity of Galaxy's synchrotron radiation $\mathrm{K}(\theta, \varphi)$ consists of an isotropic and anisotropic parts:

$$
K(\theta, \varphi)=K_{0}(\theta)+K_{2}(\theta) \cos ^{2} \varphi
$$

where $\theta$ is the angle between the lines of sight and $\varphi$ is the angle setting the direction of the regular field component normal to the line of sight $H$. The 
appearance of the anisotropic part is caused by the regular magnetic field and it is possible to find the direction of $\mathrm{H}$ by an abalysis of correlation function of synchrotron intensity. Our research [3] has shown that the expression for $K_{2}(\theta)$ can be transformed into Abel's equation and thus the reversed task can be solved for $K_{2}(\theta)$. Correlation functions of the random magnetic field as well spectrum $\mathrm{E}(\mathrm{k})$ are available:

$$
\mathrm{E}(\mathrm{k})=-\frac{\chi}{4 \pi} \mathrm{kR} \int_{0}^{\theta_{\max }} \mathrm{d} \theta \frac{\mathrm{d}^{2}}{\mathrm{~d} \theta^{2}}\left[\mathrm{~K}_{2}(\theta) \cdot \theta^{2}\right] \cdot \mathrm{J}_{1}(\mathrm{kR} \theta)
$$

where $J_{1}(x)$ is Bessel function, $\chi$ is a coefficient, $\mathrm{R}$ is the size of emmiting region. This size can be found using HII regions as distance indicators in the framework of the cross-correlation method [3].

\section{Results}

According to observational data ([4], [5]) the hierarchical structure of the Galaxy's interstellar magnetic fields can be described as follows: regular field with the intensity comparable with square root of the dispersion of the random field exists. The random magnetic field is formed by the hierarchy of turbulent magnetic vertices. The characteristic angular scale of the turbulence discussed is

about $3^{\circ}$. In order to estimate the corresponding linear scale $\mathrm{L}$ we have to know the effective depth of the emission layer. The preparations for the measurements of this value using cross-correlation method are under way at the decameter radiotelescope in vicinity of Kharkov. For the rough estimate we can take $1 \mathrm{kpc}$ as synchrotron-emissivity depth. In this case we would then obtain L 50 pc as the size of typical magnetic inhomogeneities.

\section{Discussion}

The initial results from the study of the intensity variations of the diffuse radiation by means of correlation functions show this approach to be promising one for investigating the Galaxy's magnetic field.

The method is progressing. Now it is clear that not only synchrotron intensity fluctuations, but also fluctuations of different parameters (polarization, distribution of molecular line's profiles etc.) can be used to find statistical characteristics of the interstellar medium [6]. This information is essential for better understanding of processes taking place in the interstellar medium, including the processes of fragmentation of molecular clouds and star formation.

\section{References}

1. Chibisov G.V., Lazarian A.L. Preprint 283, Moscow, FIAN, 1987.

2. Chibisov G.V., Ptuskin V.S. Proc. 17-th Int. Cosmic Ray Conf. (Paris) 2 , 233 (1981).

3. Chibisov G.V., Lazarian A.L. Preprint 191, Moscow, FIAN, 1989.

4. Oagkesamanskii R.D., Shutenkov V.R. (1987) "Radio background fluctuations and the structure of the galactic magnetic field". Sov. Astron. Lett. $\underline{13}$ (2), $73-76$.

5. Lazarian A.L., Shutenkov V.R., Preprint 180, Moscow, FIAN, 1989.

6. Lazarian A.L., Preprint 190, Moscow, FIAN, 1989. 